\title{
Generalized modeling of ecological population dynamics
}

\author{
Justin D. Yeakel · Dirk Stiefs • Mark Novak • \\ Thilo Gross
}

Received: 1 October 2010 / Accepted: 13 January 2011 / Published online: 12 February 2011

(C) The Author(s) 2011. This article is published with open access at Springerlink.com

\begin{abstract}
Over the past 7 years, several authors have used the approach of generalized modeling to study the dynamics of food chains and food webs. Generalized models come close to the efficiency of random matrix models, while being as directly interpretable as conventional differential-equation-based models. Here, we present a pedagogical introduction to the approach of generalized modeling. This introduction places more emphasis on the underlying concepts of generalized modeling than previous publications. Moreover, we propose a shortcut that can significantly accelerate the formulation of generalized models and introduce an iterative procedure that can be used to refine existing generalized models by integrating new biological insights.
\end{abstract}

\footnotetext{
J. D. Yeakel

Department of Ecology and Evolutionary Biology,

University of California, 1156 High Street,

Santa Cruz, CA 95064, USA

e-mail: jdyeakel@gmail.com

D. Stiefs

Max-Planck Institute for the Physics of Complex Systems,

Nöthnitzer Str. 38, 01187 Dresden, Germany

e-mail: stiefs@pks.mpg.de

M. Novak

Long Marine Lab, 100 Shaffer Road,

Santa Cruz, CA 95060, USA

e-mail: mnovak1@ucsc.edu

T. Gross $(\bowtie)$

Center for Dynamics, Dresden and Max-Planck Institute

for the Physics of Complex Systems,

Nöthnitzer Str. 38, 01187 Dresden, Germany

e-mail: thilo.gross@physics.org,gross@pks.mpg.de
}

Keywords Omnivory • Generalized modeling • Bifurcation - Food chain • Food web • Predator-prey system $\cdot$ Intraguild predation

\section{Introduction}

Ecological systems are fascinating because of their complexity. Not only do ecological communities harbor a multitude of different species, but even the interaction of just two individuals can be amazingly complex. For understanding ecological dynamics, this complexity poses a considerable challenge. In conventional mathematical models, the dynamics of a system of interacting species are described by a specific set of ordinary differential equations (ODEs). Because these equations are formulated on the level of the population, all complexities arising in the interaction of individuals must be cast into specific functional forms. Indeed, several important works in theoretical ecology present derivations of functional forms that include certain types of individual-level effects (Holling 1959; Rosenzweig 1971; Berryman 1981; Getz 1984; Fryxell et al. 2007). Although these allow for a much more realistic representation than, say, simple mass-action models, they cannot come close to capturing all the complexities existing in the real system. Even if detailed knowledge of the interactions among individuals were available and could be turned into mathematical expressions, these would arguably be too complex to be conducive to a mathematical analysis. In this light, the functional forms that are commonly used in models can be seen as a compromise, reflecting the aim of biological realism, 
the need to keep equations simple, and often the lack of detailed information.

Because of the many unknowns that exist in ecology, it is desirable to obtain results that are independent of the specific functional forms used in the model. This has been achieved by a number of studies that employed general models, in which at least some functional forms were not specified (Gardner and Ashby 1970; May 1972; DeAngelis et al. 1975; Murdoch and Oaten 1975; Levin 1977; Murdoch 1977; Wollkind et al. 1982). These works considered not specific models, but rather classes of models comprising simple, commonly used, functions, as well as the whole range of more complex alternatives.

That ecological systems can be analyzed without restricting the interactions between populations to specific functional forms is in itself not surprising-in every mathematical analysis the objects that are analyzed can be treated as unknown. The results of the analysis will then depend on certain properties of the unknown objects. In a general ecological model we thus obtain results that link dynamical properties of the model, e.g., the presence of predator-prey oscillations to properties of the (unknown) functions describing certain processes, e.g., the slope of the functional response evaluated at a certain point. Accordingly, the analysis of general models reveals the decisive properties of the functional forms that have a distinctive impact on the dynamics. Whether such results are ecologically meaningful depends crucially on our ability to attach an ecological interpretation to the decisive properties that are identified.

In the present paper, we specifically consider the approach called generalized modeling. This approach constitutes a procedure by which the local dynamics in models can be analyzed in such a way that the results are almost always interpretable in the context of the application. Generalized modeling was originally developed for studying food chains (Gross and Feudel 2004; Gross et al. 2004, 2005) and was only later proposed as a general approach to nonlinear dynamical systems (Gross and Feudel 2006). Subsequently, generalized modeling was used in systems biology, where it is sometimes called structural-kinetic modeling (Steuer et al. 2006, 2007; Zumsande and Gross 2010; Reznik and Segrè 2010) and is covered in recent reviews (Steuer 2007; Sweetlove et al. 2008; Jamshidi and Palsson 2008; Steuer and Junker 2009; Rodriguez and Infante 2009; Schallau and Junker 2010). In ecology, generalized models have been employed in several recent studies (Baurmann et al. 2007; van Voorn et al. 2007; Gross et al. 2009; Gross and Feudel 2009; Stiefs et al. 2010), for instance for exploring the effects of food quality on producer-grazer systems (Stiefs et al. 2010) and for identifying stabilizing factors in large food webs (Gross et al. 2009). The latter work demonstrated that the approach of generalized modeling can be applied to large systems comprising 50 different species and billions of food web topologies.

In the present paper, we present a pedagogical introduction to generalized modeling and explain the underlying idea on a deeper level than previous publications. Furthermore, we propose some new techniques that considerably facilitate the formulation and analysis of generalized models. The approach is explained using a series of ecological examples of increasing complexity, including a simple model of omnivory that has so far not been analyzed by generalized modeling.

We start out in "Local analysis of dynamical systems" with a brief introduction to fundamental concepts of dynamical systems theory. Readers who are familiar with bifurcations may wish to move directly to "Density-dependent growth of a single species", where we introduce generalized modeling by considering the example of a single population. In contrast to previous generalized analyses of this system, we use a shortcut that accelerates the formulation of generalized models. An alternative derivation is used in Section "Predator-prey dynamics", where we apply generalized modeling to a predator-prey system. Our final example, shown in "Intraguild predation", is a simple omnivory scenario involving three species. This example already contains all of the difficulties that are encountered in the analysis of larger food webs.

\section{Local analysis of dynamical systems}

Generalized modeling builds on the tools of nonlinear dynamics and dynamical systems theory. Specifically, information is typically extracted from generalized models by a local bifurcation analysis. Mathematically speaking, a bifurcation is a qualitative transition in the long-term dynamics of the system, such as the transition from stationary (equilibrium) to oscillatory (cyclic) long-term dynamics. The corresponding critical parameter value at which the transition occurs is called the bifurcation point. In this section, we review the basic procedure for locating bifurcation points in systems of coupled ODEs. This analysis is central to the exploration of both generalized and conventional models and is also covered in many excellent text books, for 
instance (Kuznetsov 2004; Guckenheimer and Holmes 2002).

In the following, we consider systems of $N$ coupled equations

$$
\frac{\mathrm{d}}{\mathrm{d} t} x_{i}=f_{i}(\mathbf{x})
$$

where $\mathbf{x}=\left(x_{1}, \ldots, x_{N}\right)$ is a vector of variables and $f(\mathbf{x})$ is a vector-valued function. In population dynamics, each $x_{i}$ typically corresponds to a population, representing the abundance, biomass, or biomass density.

The simplest form of long-term behavior that can be observed in systems of ODEs is stationarity. In a steady state $\mathbf{x}^{*}$ the right-hand side of the equations of motion vanishes,

$$
\frac{\mathrm{d}}{\mathrm{d} t} x_{i}^{*}=0
$$

for all $i$. Therefore, a system that is placed in a steady state will remain at rest for all time.

Stationarity alone does not imply that a state is a stable equilibrium. A system that is perturbed slightly from the steady state may either return to the steady state asymptotically in time or depart from the steady state entirely. For deciding whether a steady state is stable against small perturbations, we consider the local linearization of the system around the steady state, which is given by the corresponding Jacobian $\mathbf{J}$, an $N \times N$ matrix with

$J_{i j}=\left.\frac{\partial}{\partial x_{j}} f_{i}(\mathbf{x})\right|_{*}$

where $\left.\right|_{*}$ indicates that the derivative is evaluated in the steady state.

Because the Jacobian is a real matrix, its eigenvalues are either real or form complex conjugate eigenvalue pairs. A given steady state is stable if all eigenvalues of the corresponding Jacobian $\mathbf{J}$ have negative real parts. When the function $f(\mathbf{x})$ is changed continuously, for instance by a gradual change of parameters on which $f(\mathbf{x})$ depends, the eigenvalues of the corresponding Jacobian change continuously as well.

Local bifurcations occur when a change in parameters causes one or more eigenvalues to cross the imaginary axis of the complex plane. In general, this happens in either of two scenarios: In the first scenario, a real eigenvalue crosses the imaginary axis, causing a saddlenode bifurcation. In this bifurcation, two steady states collide and annihilate each other. If the system was residing in one of the steady states before the transition, the variables typically change rapidly while the system approaches some other attractor. In ecology crossing a saddle-node bifurcation backwards can, for instance, mark the onset of a strong Allee effect. In this case, one of the two steady states emerging from the bifurcation is a stable equilibrium, whereas the other is an unstable saddle, which marks the tipping point between longterm persistence and extinction.

In the second scenario, a complex conjugate pair of eigenvalues crosses the imaginary axis, causing a Hopf bifurcation. In this bifurcation, the steady state becomes unstable and either a stable limit cycle emerges (supercritical Hopf) or an unstable limit cycle vanishes (subcritical Hopf). The supercritical Hopf bifurcation marks a smooth transition from stationary to oscillatory dynamics. A famous example of this bifurcation in biology is found in the Rosenzweig-MacArthur model (Rosenzweig and MacArthur 1963), where enrichment leads to destabilization of a steady state in a supercritical Hopf bifurcation. By contrast, the subcritical Hopf bifurcation is a catastrophic bifurcation after which the system departs rapidly from the neighborhood of the steady state.

In addition to the generic local bifurcation scenarios, discussed above, degenerate bifurcations can be observed if certain symmetries exist in the system. In many ecological models, one such symmetry is related to the unconditional existence of a steady state at zero population densities. If a change of parameters causes another steady state to meet this extinct state, then the system generally undergoes a transcritical bifurcation in which the steady states cross and exchange their stability. The transcritical bifurcation is a degenerate form of the saddle-node bifurcation and is, like the saddle-node bifurcation, characterized by the existence of a zero eigenvalue of the Jacobian. Although we assume that the steady state $X^{*}$ under consideration is positive we shows in Section "Predator-prey dynamics" that the generalized analysis can include transcritical bifurcations as limit cases.

\section{Density-dependent growth of a single species}

In this section, we demonstrate how the approach of generalized modeling can be used to find local bifurcations in general ecological models. We start with the simplest example: the growth of a single-population $X$. A generalized model describing this type of system can be written as

$$
\frac{\mathrm{d}}{\mathrm{d} t} X=S(X)-D(X)
$$


where $X$ denotes the biomass or abundance of population $X, S(X)$ models the intrinsic gain by reproduction, and $D(X)$ describes the loss due to mortality. In the following we do not restrict the functions $S(X)$ and $D(X)$ to specific functional forms.

We consider all positive steady states in the whole class of systems described by Eq. 4 and ask which of those states are stable equilibria. For this purpose we denote an arbitrary positive steady state of the system as $X^{*}$. We emphasize that $X^{*}$ is not a placeholder for any specific steady state that will later be replaced by numerical values, but should rather be considered a formal surrogate for every positive steady state that exists in the class of systems.

For finding the decisive factors governing the stability of $X^{*}$, we compute the Jacobian

$\mathbf{J}^{*}=\left.\frac{\partial S}{\partial X}\right|_{*}-\left.\frac{\partial D}{\partial X}\right|_{*}$.

Because evaluated in the steady state, the two terms appearing on the right-hand side of this equation are no longer functions but constant quantities. We could therefore formally consider these terms as unknown parameters. While mathematically sound, parameterizing the Jacobian in this way leads to parameters that are hard to interpret in the context of the model and are therefore not conducive to an ecological analysis. We therefore take a slightly different approach and use the identity

$$
\left.\frac{\partial F}{\partial X}\right|_{*}=\left.\frac{F^{*}}{X^{*}} \frac{\partial \log F}{\partial \log X}\right|_{*},
$$

where $F$ is an arbitrary positive function and we abbreviated $F\left(X^{*}\right)$ by $F^{*}$. The identity, Eq. 6, holds for all $F^{*}>0$ and $X^{*}>0$; its derivation is shown in Appendix 1. Substituting the identity into the Jacobian, we obtain

$\mathbf{J}^{*}=\frac{S^{*}}{X^{*}} s_{\mathrm{x}}-\frac{D^{*}}{X^{*}} d_{\mathrm{x}}$

where

$s_{\mathrm{X}}:=\left.\frac{\partial \log S}{\partial \log X}\right|_{*}$,

$d_{\mathrm{x}}:=\left.\frac{\partial \log D}{\partial \log X}\right|_{*}$.

We note that $S^{*} / X^{*}$ and $D^{*} / X^{*}$ denote per-capita gain and loss rates, respectively. Because the gain and loss have to balance in the steady state we can define

$\alpha:=\frac{S^{*}}{X^{*}}=\frac{D^{*}}{X^{*}}$.
The parameter $\alpha$ can be interpreted as a characteristic timescale of the population dynamics. If $X$ measures abundance then this timescale is the per-capita mortality rate or in other words, the inverse of an individual's life expectancy. If $X$ is defined as a biomass then $\alpha$ denotes the biomass turnover rate. Using $\alpha$ the Jacobian can be written as

$\mathbf{J}^{*}=\alpha\left(s_{\mathrm{x}}-d_{\mathrm{x}}\right)$.

Let us now discuss the interpretation of the parameters $s_{\mathrm{x}}$ and $d_{\mathrm{x}}$. For this purpose, note that these parameters are defined as logarithmic derivatives of the original functions. Such parameters are also called elasticities, because they provide a nonlinear measure for the sensitivity of the function to variations in the argument. For any power-law $a X^{p}$ the corresponding elasticity is $p$. For instance, all constant functions have an elasticity of 0 , all linear functions an elasticity of 1 , and all quadratic functions have an elasticity of 2 . This also extends to decreasing functions such as $a / X$ for which the corresponding elasticity is -1 . For more complex functions, the value of the elasticity can depend on the location of the steady state. However, even in this case the interpretation of the elasticity is intuitive. For instance, the Holling type-II functional response is linear for low prey density and saturates for high prey density (Holling 1959). The corresponding elasticity is approximately 1 in the linear regime, but asymptotically decreases to 0 as the predation rate approaches saturation. A similar comparison for the Holling type-III function is shown in Fig. 1.

Elasticities are used in several scientific disciplines because they are directly interpretable and can be easily estimated from data (Fell and Sauro 1985). In particular, we emphasize that elasticities are defined in the state that is observed in the system under consideration, and thus do not require reference to unnatural situations, such as half-maximum values or rates at saturation that often cannot be observed directly. We note that in previous publications the elasticities have sometimes been called exponent parameters and have been obtained by a normalization procedure. In comparison to this previous procedure, the application of Eq. 6, proposed here, provides a significant shortcut.

We now return to the discussion of the example system. So far, we have managed to express the Jacobian determining the stability of all steady states by the three parameters $\alpha, s_{\mathrm{x}}$, and $d_{\mathrm{x}}$. Because this simple example contains only one variable, the Jacobian is a 1-by-1 matrix. Therefore, the Jacobian has only one eigenvalue which is directly

$\lambda=\alpha\left(s_{\mathrm{X}}-d_{\mathrm{x}}\right)$. 


\section{a}

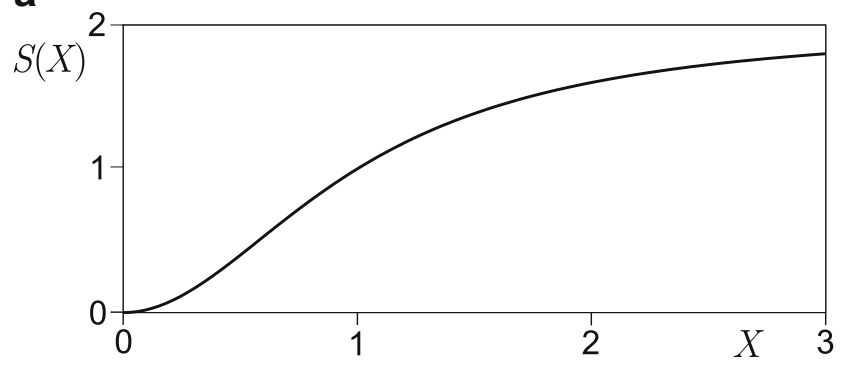

b

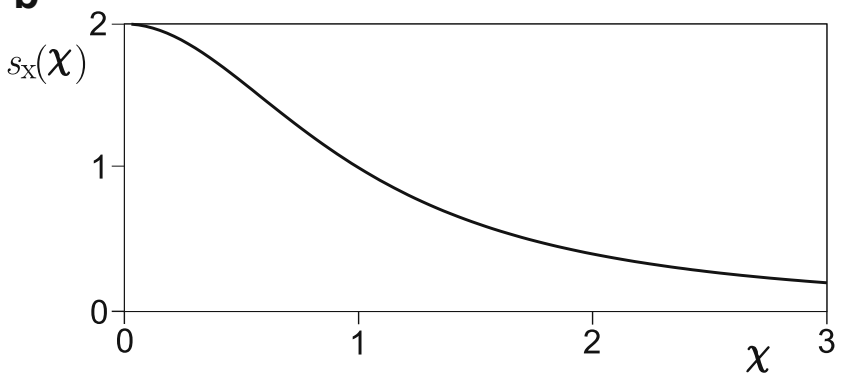

Fig. 1 Illustration of elasticity. a In the specific example system, a reproduction rate, $S(X)$ of the form of a Holling type-III functional response, $a X^{2} /\left(k^{2}+X^{2}\right)$, is assumed. This function starts out quadratically at low values of the population density $X$, but saturates as $X$ increases. b The corresponding elasticity, $s_{\mathrm{X}}$, is close to two near the quadratic regime $\chi=X^{*} / k \approx 0$, but approaches zero as saturation sets in

The steady state under consideration is stable if $\lambda<0$, or equivalently

$s_{\mathrm{X}}<d_{\mathrm{x}}$.

In words: In every system of the form of Eq. 4, a given steady state is stable whenever the elasticity of the mortality in the steady state exceeds the elasticity of reproduction.

A change in stability occurs when the elasticities of gain and loss become equal,

$s_{\mathrm{X}}=d_{\mathrm{x}}$.

If this occurs the eigenvalue of the Jacobian vanishes and the system undergoes a saddle-node bifurcation.

For gaining a deeper understanding of how the generalized analysis relates to conventional models, it is useful to consider a specific example. We emphasize that this step is not part of the analysis of the generalized model, but is presented here solely for the purpose of illustration. One model that immediately comes to mind is logistic growth, which can formally be written as a linear reproduction and quadratic mortality. However, based on our discussion above, it is immediately apparent that linear reproduction must correspond to $s_{\mathrm{x}}=1$ and quadratic mortality to $d_{\mathrm{x}}=2$. Without further analysis we can therefore say that steady states found for a single population under logistic growth must always be stable regardless of the other parameters.

A more interesting example is obtained when one assumes a reproduction rate following a Holling typeIII kinetic and linear mortality,

$\frac{\mathrm{d}}{\mathrm{d} t} X=\frac{a X^{2}}{k^{2}+X^{2}}-b X$,

where $a$ is the growth rate at saturation, $k$ is the halfsaturation value of growth, and $b$ is the mortality rate. This example system can be investigated by explicit computation of steady states and subsequent stability and bifurcation analysis. This procedure is shown in most textbooks on mathematical ecology and is hence omitted here. For the present example the conventional analysis reveals that, for high $k$ only a trivial equilibrium at zero population density exists, so that the population becomes extinct deterministically (Fig. 2a). As $k$ is reduced, a saddle-node bifurcation occurs, which marks the onset of a strong Allee effect. In the bifurcation, a stable nontrivial equilibrium and an unstable saddle point are created. Beyond the bifurcation a population can persist if its initial abundance is above the saddle point. In this case, the population asymptotically approaches the stable equilibrium. By contrast, a population which is initially below the saddle point declines further and approaches the trivial (extinct) equilibrium.

For comparing the results from the specific analysis to the generalized model, we compute the elasticities that characterize the steady states found in the specific model. Because the mortality rate is assumed to be linear, we know $d_{\mathrm{x}}=1$. The elasticity of the growth function can be found by applying Eq. 6 to the known growth function of the specific model. This yields

$s_{\mathrm{X}}=\frac{2}{1+\chi^{2}}$

where $\chi=X^{*} / k$. A detailed derivation of this relationship using a normalization procedure instead of the shortcut, Eq. 6, is given in Gross et al. (2004). Equation 16 shows that the elasticity of growth is $s_{\mathrm{X}} \approx 2$ for $X^{*} \ll k$, but approaches $s_{\mathrm{X}}=0$ in the limit $X^{*} \gg k$ (Fig. 1).

In Fig. 2a, we have color coded the growth elasticity of steady states visited by the system as $k$ is changed. We note that the saddle-node bifurcation occurs at $s_{\mathrm{X}}=$ $d_{\mathrm{x}}=1$, conforming to our expectation from the generalized model. Moreover, in the unstable saddles we find 


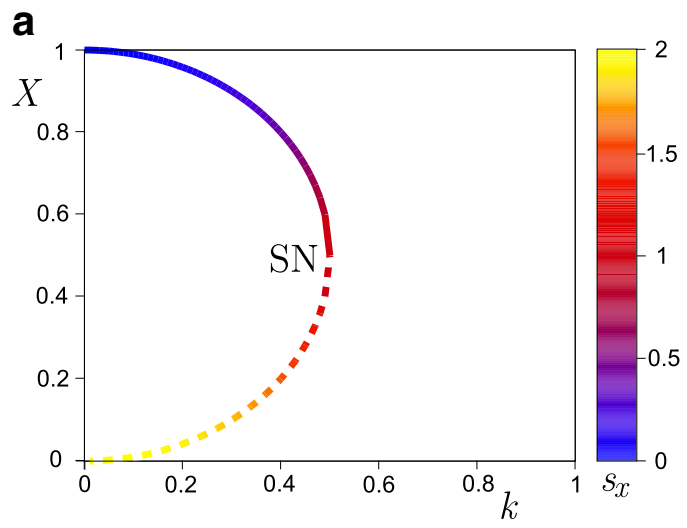

Fig. 2 Comparison of generalized and conventional modeling. a Bifurcation diagram of a specific example (Eq. 15). The lines correspond to the locations of steady states, which are stable equilibria (solid) or saddles (dashed). The color encodes the elasticity of growth, $s_{\mathrm{X}}$, in the respective steady states. The figure confirms the our expectation from the generalized model that steady states are stable whenever $s_{\mathrm{X}}<d_{\mathrm{X}}$, where $d_{\mathrm{x}}=1$ in the specific example. The two steady states vanish in a saddle-node

$s_{\mathrm{x}}>d_{\mathrm{x}}$, whereas the stable equilibria are characterized by $s_{\mathrm{X}}<d_{\mathrm{X}}$, which is in agreement with Eq. 13 .

We can now map the steady states found in the specific model into the generalized parameter plane spanned by the elasticities $s_{\mathrm{X}}$ and $d_{\mathrm{x}}$ (Fig. 2b). Because $d_{\mathrm{x}}=1$ in the specific example, irrespective of $X^{*}$, all steady states end up on a single line in the generalized diagram. Other areas of the bifurcation diagram, not visited by the specific example, correspond to other models that assume other functional forms for the mortality. In this diagram the two colliding branches of stable and unstable steady states are mapped into the corresponding stable and unstable region of the generalized parameter space, respectively. Therefore the two branches appear on different sides of the bifurcation. However, from the bifurcation condition, Eq. 14, we know that this bifurcation must occur as the diagonal line in the diagram is crossed.

The comparison of the two bifurcation diagrams in Fig. 2 highlights the differences between generalized and conventional modeling. In the conventional model different numbers of steady states are found depending on the specific functional forms that are assumed. Moreover, for a given set of parameter values multiple steady states can coexist that differ in their stability properties. Because the generalized model comprises a whole class of specific models a single set of generalized parameters corresponds to an infinite number of different steady states, found in different specific models. However, the solution branches of this family of models have been unfolded such that all steady states

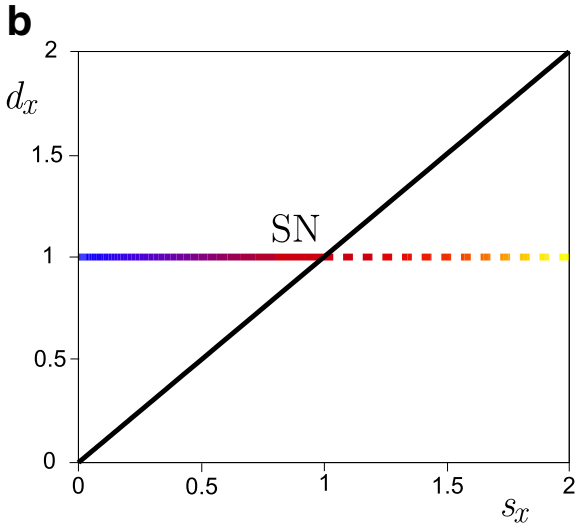

bifurcation, which occurs at $s_{\mathrm{X}}=d_{\mathrm{x}}$. Parameters: $a=b=1$. b General results. The correspondence between generalized and specific model can be seen explicitly by mapping the steady states from the specific model into the generalized parameter plane. In this plane, the stable and unstable states are separated by the stability boundary (black line, Eq. 14) at which the saddle-node bifurcation occurs

corresponding to the same set of generalized parameters must have the same stability properties.

It is apparent that for a given specific example, the conventional analysis reveals more detailed insights than the generalized analysis. For instance the presence of the strong Allee effect that is directly evident in the conventional bifurcation diagram, Fig. 2a, can only be inferred indirectly from the presence of the saddlenode bifurcation in the generalized analysis, Fig. $2 b$. However, the conventional analysis provides insights only into the dynamics of the specific model under consideration, whereas the generalized analysis reveals the stability boundary (black line in Fig. 2b) that is valid for the whole class of models and is hence robust against uncertainties in the specific model.

A major advantage of the generalized model is that results are obtained without explicit computation of steady states. In the conventional model that we discussed in this section, steady states can be computed analytically. However, even for slightly more complex models this computation becomes infeasible as it involves (under the best circumstances) factorization of large polynomials. Also the numerical computation of steady states poses a serious challenge for which no algorithm with guaranteed convergence is known. Because generalized modeling avoids the explicit computation of steady states, the approach can be scaled to much larger networks. The additional complications that arise in the generalized modeling of larger systems and their resolution are the subject of the subsequent sections. 


\section{Predator-prey dynamics}

In our second example, we consider a slightly more complex system where intra- and interspecific interactions are considered. Departing from the single-species model, we introduce a predator $Y$ whose growth is entirely dependent on $X$. This leads to the generalized model

$$
\begin{aligned}
\frac{\mathrm{d}}{\mathrm{d} t} X & =S(X)-D(X)-F(X, Y), \\
\frac{\mathrm{d}}{\mathrm{d} t} Y & =\gamma F(X, Y)-M(Y),
\end{aligned}
$$

where $S(X)$ and $D(X)$ describe the reproduction and mortality of the prey $X$, the function $F(X, Y)$ models the interaction of $X$ with the predator $Y, M(Y)$ is the mortality of $Y$, and $\gamma$ is a constant conversion efficiency.

In principle this model can be analyzed by the procedure proposed in the previous section. However, for gaining a different perspective we use the alternative procedure for deriving Jacobians that was used in previous papers on generalized modeling, such as Gross and Feudel (2006). This alternative procedure starts by introducing a set of normalized variables

$x=\frac{X}{X^{*}}$

$y=\frac{Y}{Y^{*}}$

and normalized functions, indicated by lower-case letters, such as

$s(x)=\frac{S(X)}{S^{*}}=\frac{S\left(x X^{*}\right)}{S^{*}}$,

where we used the abbreviated notation $S^{*}:=S\left(X^{*}\right)$. Using these definitions we can rewrite Eqs. 17, 18 as

$$
\begin{aligned}
& \frac{\mathrm{d}}{\mathrm{d} t} x=\frac{S^{*}}{X^{*}} s(x)-\frac{D^{*}}{X^{*}} d(x)-\frac{F^{*}}{X^{*}} f(x, y), \\
& \frac{\mathrm{d}}{\mathrm{d} t} y=\gamma \frac{F^{*}}{Y^{*}} f(x, y)-\frac{M^{*}}{Y^{*}} m(y) .
\end{aligned}
$$

By normalizing the system we have mapped the previously unknown steady state $\left(X^{*}, Y^{*}\right)$ to a known loca- tion $\left(x^{*}, y^{*}\right)=(1,1)$. Linearizing the system around this steady state, we obtain the Jacobian

$\mathbf{J}^{*}=\left(\begin{array}{cc}\frac{S^{*}}{X^{*}} s_{\mathrm{x}}-\frac{D^{*}}{X^{*}} d_{\mathrm{x}}-\frac{F}{X^{*}} f_{\mathrm{x}} & -\frac{F^{*}}{X^{*}} f_{\mathrm{y}} \\ \gamma \frac{F^{*}}{Y^{*}} f_{\mathrm{x}} & \gamma \frac{F^{*}}{Y^{*}} f_{\mathrm{y}}-\frac{M^{*}}{Y^{*}} m_{\mathrm{y}}\end{array}\right)$,

where we again used roman indices to indicate partial derivatives, $s_{\mathrm{X}}=\partial s(x) /\left.\partial x\right|_{*}$. As suggested by this notation the partial derivatives of the normalized functions are logarithmic derivatives (i.e., elasticities) of the original functions. For instance

$s_{\mathrm{X}}:=\left.\frac{\partial \log S}{\partial \log X}\right|_{*}$

We now continue in analogy to the previous section. We absorb the steady-state abundances $X^{*}, Y^{*}$, rates $S^{*}, D^{*}, F^{*}, M^{*}$, and the constant $\gamma$ into a set of scale parameters. In doing so, we have to take care to satisfy the demands of stationarity for every variable. Let us first consider the predator. The corresponding equation of motion, Eq. 18, implies

$\gamma F^{*}-M^{*}=0$.

Analogously to the example from the previous section, we can therefore define

$\alpha_{y}:=\gamma \frac{F^{*}}{Y^{*}}=\frac{M^{*}}{Y^{*}}$.

which automatically satisfies Eq. 26 but does not restrict the per-capita rates otherwise. As in the previous example, the $\alpha_{y}$ can be interpreted as a characteristic time scale, now describing the predator population.

Equation 27 allows us to replace all occurrences of the unknown constants from the elements of the Jacobian referring to the predator, i.e., the bottom row in Eq. 24. This is possible because the equation of motion for the predator, Eq. 18, contains only two terms and can therefore be characterized by two rates, the reproduction rate and the mortality rate. By considering steady states we impose one constraint, Eq. (26). Thus, only one degree of freedom remains, which can be captured by one parameter $\alpha_{y}$.

An additional complication is encountered for the prey. Because we modeled predation and mortality from intraspecific competition as independent loss terms, the corresponding equation of motion, Eq. 17 contains three terms. Demanding stationarity yields the constraint

$S^{*}-D^{*}-F^{*}=0$. 
The presence of three terms, subject to one constraint, implies that two generalized parameters have to be defined to replace all occurrences of the unknown rates. From the point of view of mathematics, many alternative ways of defining these parameters exist. However, from an ecological point of view these ways differ in the interpretability of the parameters they introduce. Analyzing generalized models we found it almost always advantageous to introduce (a) one parameter capturing the characteristic timescale of the corresponding variable, i.e., the turnover rate, (b) a set of branching parameters capturing the relative contribution of the individual loss terms to the total turnover, (c) a set of branching parameters capturing the relative contribution of individual gain terms to the total turnover.

By definition, the turnover rate (step a) equals the sum of all gains and the sum of all losses. In the present example, we thus define

$\alpha_{x}:=\frac{S^{*}}{X^{*}}=\frac{D^{*}}{X^{*}}+\frac{F^{*}}{X^{*}}$,

where all gains appear on the left side of the equals sign and all losses appear on the right. In step (b), we define the parameter

$\beta:=\frac{1}{\alpha_{x}} \frac{D^{*}}{X^{*}}$,

and its complement

$\bar{\beta}:=\frac{1}{\alpha_{x}} \frac{F^{*}}{X^{*}}$,

that capture the relative contribution of losses from predation and intraspecific competition to the total turnover rate. Because the losses have to add up to the total turnover, the parameters have to obey $\beta+\bar{\beta}=1$. Therefore, only one of the two parameters, say $\beta$, can be considered as an independent parameter. Because there is only one gain term, step (c) in the outline above is not necessary in the present example.

As we already argued above, we obtained two independent parameters describing the biomass flow in the prey population: the per-capita turnover rate, $\alpha$, and the relative contribution of intraspecific competition to the total turnover rate $\beta$, i.e., the fraction of losses caused by competition.

In general, the same strategy for defining branching parameters can be applied to equations containing any number of terms. For each variable, firstly define a parameter $\alpha$, which denotes the total turnover rate, separating gain and loss terms, and identifying the characteristic timescale of a species. Branching parameters are then assigned to any number of terms that define the relative contribution of the individual gains and losses to the total turnover within a system.

Returning to the Jacobian of the predator-prey system, we substitute the scale and branching parameters into Eq. 24, which yields

$\mathbf{J}^{*}=\left(\begin{array}{cc}\alpha_{x}\left(s_{\mathrm{x}}-\beta d_{\mathrm{x}}-\bar{\beta} f_{\mathrm{x}}\right) & -\alpha_{x} \bar{\beta} f_{\mathrm{y}} \\ \alpha_{y} f_{\mathrm{x}} & \alpha_{y}\left(f_{\mathrm{y}}-m_{\mathrm{y}}\right)\end{array}\right)$.

In contrast to the system from the previous section, the Jacobian is now a 2-by-2 matrix. For this Jacobian, the eigenvalues can still be computed analytically. However, analytical eigenvalue computation is tedious already for systems with three variables, and in general impossible for systems with more than four variables. Nevertheless, analytical results can be obtained even for larger systems by deriving test functions that directly test for bifurcations, without an intermediate computation of eigenvalues.

Saddle-node bifurcations occur when a single real eigenvalue crosses the imaginary axis (Kuznetsov 2004). Therefore, a zero eigenvalue must be present in a saddle-node bifurcation. This implies that the product of all eigenvalues must vanish in this bifurcation. Because the product of all eigenvalues equals the determinant of a matrix, we can locate saddle-node bifurcations by demanding that the determinant of the Jacobian, $\operatorname{det} \mathbf{J}^{*}$, vanishes. For the present example this yields the condition

$s_{\mathrm{x}}=\frac{\left(\beta d_{\mathrm{x}}+\bar{\beta} f_{\mathrm{x}}\right)\left(f_{\mathrm{y}}-m_{\mathrm{y}}\right)-\bar{\beta} f_{\mathrm{x}} f_{\mathrm{y}}}{f_{\mathrm{y}}-m_{\mathrm{y}}}$.

For finding the Hopf bifurcations, we note that the trace of a matrix (the sum of diagonal elements) is identical to the sum of the eigenvalues (Kuznetsov 2004). For a two-dimensional system this implies that the trace of the Jacobian, tr $\mathbf{J}^{*}$, must vanish in a Hopf bifurcation, because there is only one purely symmetric eigenvalue pair, which adds up to zero. For detecting Hopf bifurcations we have to additionally demand that the det $\mathbf{J}^{*}>0$, because $\operatorname{tr} \mathbf{J}^{*}=0$ is also satisfied if there is a real symmetric pair of eigenvalues, which is not characteristic of the Hopf bifurcation. In the predatorprey model, the Hopf bifurcation is found at

$s_{\mathrm{x}}=\beta d_{\mathrm{x}}+\bar{\beta} f_{\mathrm{x}}-r\left(f_{\mathrm{y}}-m_{\mathrm{y}}\right)$,

where $r=\alpha_{y} / \alpha_{x}$ is the turnover rate of the predator measured in multiples of the turnover rate of the prey. For systems with more than two variables, the test function for the Hopf bifurcation can be derived by a procedure that is described in Gross and Feudel (2004).

The results of the bifurcation analysis suggest that high values of $f_{\mathrm{x}}$ exert a stabilizing influence on the system. Previous studies (Gross et al. 2004; Stiefs et al. 
2010) showed that this parameter is relevant for enrichment scenarios. In many previously proposed models, predator saturation increases when resources are added, leading to a decrease of $f_{\mathrm{x}}$ and therefore to instability. Identification of $f_{\mathrm{x}}$ as a crucial parameter for stability in the generalized model enables us to ask what functional responses would lead to an intermediate stabilizing effect of enrichment that is sometimes observed in nature. The discussion in Gross et al. (2004) showed that reasonable functional responses can be found that exhibit such an intermediate stabilization, but are very hard to distinguish from, say Holling typeII kinetics, if they were encountered in nature.

To illustrate the differences between generalized and conventional modeling, we again compare the generalized model with a specific example. For this purpose we focus on the Rosenzweig-MacArthur model. In this model, the prey exhibits logistic growth in absence of the predator, the predator-prey interaction is modeled by a Holling type-II functional response, and the mortality of the predator is assumed to be density independent. This leads to

$$
\begin{aligned}
& \frac{\mathrm{d}}{\mathrm{d} t} X=r X\left(1-\frac{X}{k}\right)-\frac{a X Y}{b+X}, \\
& \frac{\mathrm{d}}{\mathrm{d} t} Y=\gamma \frac{a X Y}{b+X}-m Y,
\end{aligned}
$$

where $r$ is the intrinsic growth rate of $X, k$ is the carrying capacity of $X, a$ is the predation rate at saturation, $b$ is the half-saturation value of the predation rate, $\gamma$ is the biomass conversion efficiency, and $m$ is the mortality rate of $Y$.

The results of a conventional bifurcation analysis are shown in Fig. 3a. If the carrying capacity $k$ is too small then the predator population cannot invade the system. As the carrying capacity is increased a transcritical bifurcation occurs in which a stable equilibrium appears, such that the predator-prey system can reside in stationarity. If the carrying capacity is increased further, a supercritical Hopf bifurcation occurs, in which the equilibrium is destabilized. Subsequently, the system resides on a stable limit cycle, which emerges from the Hopf bifurcation. On this cycle, pronounced predatorprey oscillations can be observed, which become larger as the carrying capacity is further increased.

One can imagine that if an additional parameter is changed then critical values of the carrying capacity at which the bifurcations occur change as well. This can be visualized in two-parameter bifurcation diagrams, which we have already used for the generalized model in Fig. 2b. In such diagrams, Hopf and saddle-node bifurcation points form lines in the two-dimensional parameter space. For the specific example of the Rosenzweig-MacArthur system, a twoparameter bifurcation diagram is shown in Fig. 3b. This diagram illustrates that increasing the mortality rate $m$ of the predator, shifts both the transcritical bifurcation point and the Hopf bifurcation point to higher values of the carrying capacity.

For comparing the specific example to the generalized model, we compute the generalized parameters that are observed in the steady states of the specific model. Above, we have already noted that logistic growth can be understood as a combination of linear reproduction and quadratic mortality, which corresponds to $s_{\mathrm{x}}=1$ and $d_{\mathrm{x}}=2$. Furthermore, the assumptions of density independent mortality and linear dependence of the predation rate on the predator imply $m_{\mathrm{y}}=$ $f_{\mathrm{y}}=1$. The elasticity $f_{\mathrm{x}}$ of the predation rate with respect to prey was derived in Gross et al. (2004) and is

$f_{\mathrm{x}}=\frac{1}{1+\chi}$,

where $\chi=X^{*} / b$. Accordingly, $f_{\mathrm{x}}=1$ in the limit of vanishing prey density and $f_{\mathrm{x}}=0$ in the limit of infinite prey. Note that in the Rosenzweig-MacArthur model, the predator population tightly controls the prey population. Once the predator can invade, any further increase in carrying capacity only increases the stationary population of the predator, while the stationary population size of the prey remains invariant.

Apart from the parameters $\beta$ and $f_{\mathrm{X}}$, shown in Fig. $3 c$, the only other parameter that is not fixed to a specific value is the relative turnover of the predator $r=\alpha_{y} / \alpha_{x}$. This parameter cannot affect the transcritical bifurcation, because turnover rates by construction cannot appear in test functions of transcritical or saddle-node bifurcations. By contrast, turnover rates in general affect Hopf bifurcations. However, in the present example the dependence of the Hopf bifurcation test function, Eq. 34 on $r$ disappears if density independent mortality and linear dependence of the predation rate on the predator population are assumed. Therefore, the parameter has no influence on the bifurcation surfaces. We note that this is a special property of the Rosenzweig-MacArthur system and not a generic feature of the larger class of systems described by the generalized model. As argued in van Voorn et al. (2007) and Gross and Feudel (2009) one can expect that typically mortality is slightly super-linear because of overcrowding, diseases and other limiting resources, whereas predation may be sublinear due to predator interference. In this case, large values of $r$ can have a stabilizing effect. 

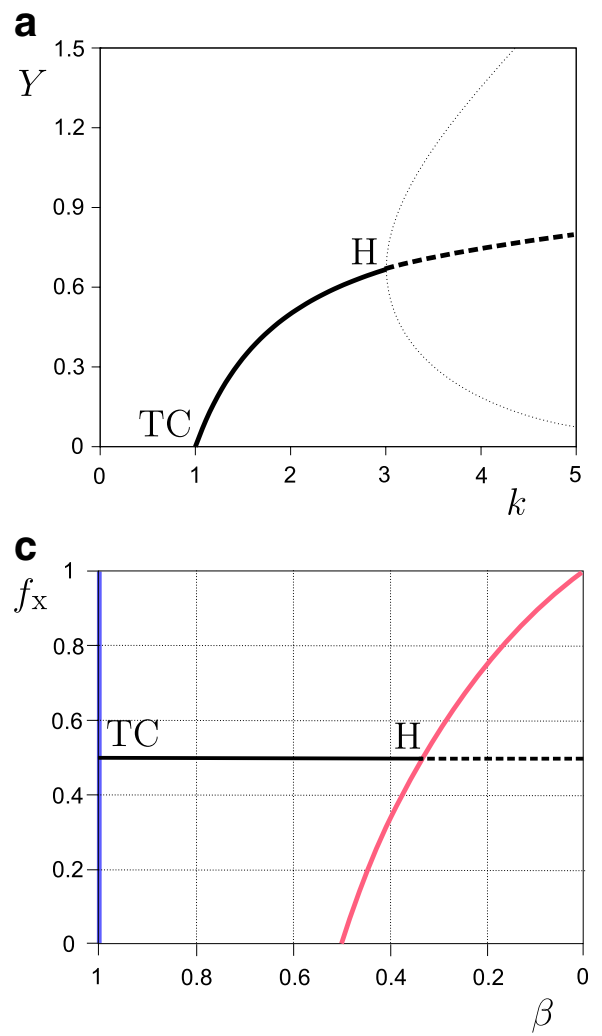

Fig. 3 Comparison of a generalized model and a specific example for predator-prey interaction. a Bifurcation diagram of the Rosenzweig-MacArthur model. The predator $Y$ can invade the system when the carrying capacity $k$ of the prey exceeds a threshold, corresponding to a transcritical bifurcation $(T C)$. Increasing the carrying capacity further eventually leads to destabilization in a Hopf bifurcation $(H)$. Lines mark stable (solid) and unstable (dashed) steady states and the upper and lower turning points of a stable limit cycle (dotted). Parameters: $r=1, a=2, \gamma=$ $0.5, b=1, m=0.5$. b A two-parameter bifurcation diagram of the Rosenzweig-MacArthur model as a function of the mortality of $Y, m$, and the carrying capacity of $X, k$. Stable equilibria are confined to the narrow region between the TC (blue) and the b

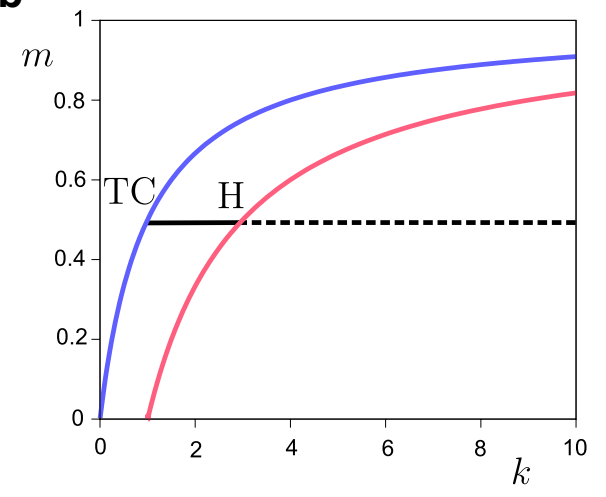

d

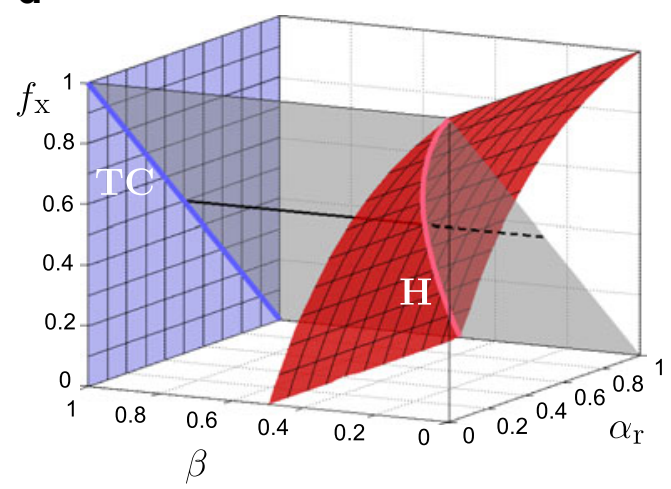

$\mathrm{H}$ (red) bifurcation points. The black lines indicate the steady states found in the section of this diagram shown in (a). c A twoparameter bifurcation diagram of the generalized predator-prey model as a function of the proportional mortality of $X$ due to intraspecific competition $(\beta)$ and the elasticity of the predation rate with respect to the prey $\left(f_{\mathrm{x}}\right)$. Bifurcations and labels are as above. The black line plots the trajectory of the RosenzweigMacArthur system as a specific example of the class of models. d A three-parameter bifurcation diagram of the generalized predator-prey model. The bifurcation points now form surfaces. The black lines indicate the steady states from (a), while the grey plane indicates all steady states that can be reached in the Rosenzweig-MacArthur model if $k$ and $m$ are varied as in (b)
We can now map the steady states to the specific system into the generalized parameter space. A twoparameter bifurcation diagram of the generalized model is shown in Fig. 3c. In this diagram, bifurcations of saddle-node type occur only on the boundary of the parameter space, where the branching parameter $\beta$ vanishes. This parameter value indicates that none of the biomass loss of the prey occurs because of predation. Even without comparing to the specific example we can conclude that this bifurcation must be a transcritical bifurcation in which the predator enters the system. To illustrate this we map additionally the twodimensional bifurcation diagram (Fig. 3b) into the generalized parameter space. This mapping is visualized in a three-dimensional bifurcation diagram shown in Fig. 3d. Such three-dimensional diagrams can be generated from analytical test functions using the method described in Stiefs et al. (2008) and have been used in a number of previous studies (Gross and Feudel 2004; Gross et al. 2004, 2005, 2009; Stiefs et al. 2010; Gross and Feudel 2009). As in the two-parameter diagrams, every point in the diagram represents a family of steady states. The parameter volume is divided by bifurcation surfaces, which separate steady states with qualitatively different local dynamics. Specifically, all steady states located between the two bifurcation surfaces are stable, whereas the steady states below the Hopf bifurcation surface are unstable. 
In the present example, we were able to show all relevant parameters in a single three-parameter bifurcation diagram. Let us remark that this is in general not possible as a larger number of parameters is often necessary to capture the dynamics of a system at the desired degree of generality. Even if a generalized model contains only five parameters, the three-dimensional slice that can be visualized in a single three-parameter diagram is relatively small when compared with the five-dimensional space. Nevertheless, plotting threeparameter bifurcation diagrams can be very valuable because a three-dimensional diagram is often sufficient to locate bifurcations of higher codimension. Such bifurcations are formed at the point in parameter space where different bifurcation surfaces meet or intersect. The presence of such bifurcations can reveal additional insights into global properties of the dynamics. For instance in Gross et al. (2005) the presence of a certain bifurcation of higher codimension in generalized models was used to show that chaotic dynamics generically exist in long food chains. An extensive discussion of bifurcations of higher codimension and their dynamical implications is presented in Kuznetsov (2004). For obtaining a general overview of the dynamics of larger systems containing hundreds or thousands of parameters, bifurcation diagrams are not suitable. However, these systems can be analyzed by statistical sampling techniques described in the following section.

\section{Intraguild predation}

As the final example in the present paper, we consider the effect of omnivory on a small food web. Omnivory is defined by an organism's ability to consume prey that inhabit multiple trophic levels. It has been the subject of much recent interest because it is notable for its pervasiveness within well-studied ecosystems (Polis 1991), as well as its relatively complex dynamics (McCann and Hastings 1997; Kuijper et al. 2003; Tanabe and Namba 2008).

Omnivory has been historically viewed as a paradoxical interaction. Initially, the presence of omnivory was thought to be entirely destabilizing, and, as a consequence, rarely observed in nature (Pimm and Lawton 1978). However, further explorations of ecological networks have reported omnivory to be a common architectural component within larger food webs (Bascompte et al. 2005; Stouffer and Bascompte 2010). Furthermore, theoretical investigations have revealed parameter regions that lead to both stabilizing and destabilizing dynamics in simple models (Holt and Polis 1997; McCann and Hastings 1997; Kuijper et al.
2003; Tanabe and Namba 2008; Namba et al. 2008; Verdy and Amarasekare 2010). These theoretical arguments are limited by the fact that such models are either constrained to specific functional forms or report dynamics across parameter ranges that may not be biologically significant. A generalization of the entire class of simple omnivory models is poised to elucidate under which conditions stable or unstable dynamics are bound to occur, regardless of the functional relationships among or between species in the model.

A specific case of omnivory is intraguild predation (IGP), which in its simplest incarnation appears in a three-species system containing a consumer and resource pair (as in the prior example), and an omnivore that predates upon both the consumer and resource. Traditional analyses of IGP models reached the following: (1) the coexistence of all species in the system is contingent on the greater competitive abilities of $Y$, relative to the omnivore $Z$, to consume the shared resource (Holt and Polis 1997; McCann and Hastings 1997); (2) enrichment destabilizes the system (Holt and Polis 1997; Diehl and Feissel 2001); (3) if the gain of the omnivore by predation on the consumer exceeds the negative competitive effects of the consumer, then the consumer facilitates a larger population of the omnivore than can be maintained in its absence (Diehl and Feissel 2001).

In the present paper, we consider the generalized model

$$
\begin{aligned}
\frac{\mathrm{d}}{\mathrm{d} t} X & =S(X)-D(X)-F(X, Y)-G(X, Y, Z) \\
\frac{\mathrm{d}}{\mathrm{d} t} Y & =\gamma F(X, Y)-H(X, Y, Z)-M(Y) \\
\frac{\mathrm{d}}{\mathrm{d} t} Z & =K(X, Y, Z)-M(Z) .
\end{aligned}
$$

In addition to the terms already presented in the predator-prey model from "Predator-prey dynamics", we included the functions $G$ and $H$, which denote the loss of the resource and consumer from predation by the omnivore and the function $K$ denoting the gain of the omnivore that arises from this predation. Note that we modeled the two different predatory losses of $X$ as separate terms $G$ and $H$ because these losses can be assumed to arise independently of each other. By contrast the gain of the omnivore derives from predation on two different prey species and is modeled as a single term $K$ because finite handling time, saturation effects, and possibly active prey-switching behavior prevent the predator from feeding on both sources independently of each other. 
By following the procedure described in the previous sections, we construct the Jacobian

$\mathbf{J}^{*}=\left(\begin{array}{ccc}\alpha_{x}\left(s_{\mathrm{x}}-\delta d_{\mathrm{x}}-\bar{\delta}\left(\beta_{x} f_{\mathrm{x}}-\bar{\beta}_{x} g_{\mathrm{x}}\right)\right) & -\alpha_{x} \bar{\delta}\left(\beta_{x} f_{\mathrm{y}}+\bar{\beta}_{x} g_{\mathrm{y}}\right) & -\alpha_{x} \bar{\beta}_{x} \bar{\delta} g_{z} \\ \alpha_{y}\left(f_{\mathrm{x}}-\beta_{y} h_{\mathrm{x}}\right) & \alpha_{y}\left(f_{\mathrm{y}}-\beta_{y} h_{\mathrm{y}}-\bar{\beta}_{y} m_{\mathrm{y}}\right) & -\alpha_{y} \beta_{y} h_{\mathrm{z}} \\ \alpha_{z} k_{\mathrm{x}} & \alpha_{z} k_{\mathrm{y}} & \alpha_{z}\left(k_{\mathrm{z}}-m_{\mathrm{z}}\right)\end{array}\right)$

where the elasticities are defined as in the previous sections and the scale parameters are

$\alpha_{x}=\frac{S^{*}}{X^{*}}=\frac{D^{*}}{X^{*}}+\frac{F^{*}}{X^{*}}+\frac{G^{*}}{X^{*}}$,

$\alpha_{y}=\gamma \frac{F^{*}}{Y^{*}}=\frac{H^{*}}{Y^{*}}+\frac{M^{*}}{Y^{*}}$,

$\alpha_{z}=\frac{K^{*}}{Z^{*}}=\frac{M^{*}}{Z^{*}}$,

the branching parameters are

$\delta=\frac{D^{*}}{D^{*}+F^{*}+G^{*}}, \quad \beta_{x}=\frac{F^{*}}{F^{*}+G^{*}}, \quad \beta_{y}=\frac{H^{*}}{H^{*}+M^{*}}$,

and $\bar{\delta}=1-\delta, \bar{\beta}_{x}=1-\beta_{x}$, and $\bar{\beta}_{y}=1-\beta_{y}$.

Let us remark that the branching parameters in the model were defined such that the parameter $\delta$ separates the predatory losses of the resource from the competition term. This was done to reflect our opinion that these losses are qualitatively different. An alternative procedure would have been to use three branching parameters, $\beta_{d}, \beta_{f}, \beta_{g}$, to denote directly the different proportions the three losses contribute to the total percapita loss rate of $X$. In this case, we would have to demand $\beta_{d}+\beta_{f}+\beta_{g}=1$ for consistency, such that only two of the parameters could be varied independently.

In principle, the Jacobian of the omnivory model could be analyzed straight away. However, more insights can be gained by building more biological knowledge into the model. In the following, we integrate this knowledge into the Jacobian derived above, by a refinement procedure that can be used to iteratively integrate new information into the generalized model when such information becomes available.

In the present example, we want to integrate the observation that the different elasticities associated with functions describing predation by the omnivore cannot be unrelated. Above, we already argued that the nonlinearity in the predator functional response arises mainly from predator saturation. If the availability of a given prey species is increased then predator saturation increases and consequently predation on other prey populations decreases. Thus saturation governs both the nonlinearity of a given predator-prey interaction and the response of the predation rate to changes in the abundance in a another prey species. For making these dependencies explicit in our model we first note that saturation depends on the total amount of prey available to the predator (see Holling 1959 for a detailed discussion). For simplicity, we assume that this total amount, $T$, is a weighted sum of sizes of the two prey populations, such that

$T(X, Y)=T_{x} X+T_{y} Y$.

We denote the relative proportions that both types of prey (here, consumer and resource) contribute to the diet of the predator (omnivore) as

$t_{x}=\frac{T_{x} X}{T(X, Y)}, \quad t_{y}=\frac{T_{y} Y}{T(X, Y)}$,

such that $t_{x}+t_{y}=1$. If a species contributes a given proportion to the diet of the omnivore, it is reasonable to assume that the same species carries an equal portion of the losses inflicted by the omnivore, such that $G(X, Y, Z) \propto t_{\mathrm{x}} K(T(X, Y), Z)$ and $H(X, Y, Z)$ $\propto t_{\mathrm{y}} K(T(X, Y), Z)$.

By considering these assumptions in the steady state under consideration and applying the identity Eq. 6 we find

$g_{\mathrm{x}}=k_{t} t_{x}+t_{y}$,

$g_{\mathrm{y}}=k_{t} t_{y}-t_{y}$

$h_{\mathrm{x}}=k_{t} t_{x}-t_{x}$,

$h_{\mathrm{y}}=k_{t} t_{y}+t_{x}$,

$k_{\mathrm{x}}=k_{t} t_{x}$,

$k_{\mathrm{y}}=k_{t} t_{y}$.

An exemplary derivation of one of the relations is shown in Appendix 2. The new parameter $k_{\mathrm{t}}$ appearing above is the elasticity of the omnivore's gain with respect to the total amount of available prey, i.e., the saturation of the omnivore. This parameter can be interpreted completely analogously to the parameter $f_{\mathrm{x}}$ in the predator-prey system.

Taking additional biological insights into account has led to relationships that can be directly substituted into 
the previously derived Jacobian. Doing so removes six parameters from the generalized model at the cost of introducing two new ones. The substitution makes the model less general and more specific, allowing us to extract more conclusions on a narrower range of models. By this procedure new insights on a given system can be integrated iteratively without re-engineering the model from scratch. We believe that such refinements will be valuable for future food web models possibly containing hundreds of species.

Let us remark that iterative refinement is not contingent on the availability of a specific, i.e., nongeneral, equation. Instead of the specific relationship in Eq. 41, we could have also used the general relationship $T(X, Y)=C_{x}(X)+C_{y}(Y)$, where $C_{x}$ and $C_{y}$ are general functions. Even substituting this general relationship into the model leads to a reduction of parameters of the model. Furthermore, the functions $C_{x}$ and $C_{y}$ can be used to introduce active prey switching. This has been done for instance in the food web models proposed in Gross and Feudel (2006) and Gross et al. (2009).

Using the techniques described above, the local bifurcations of the IGP model can be calculated analytically. However, because the number of parameters is relatively large, even three-parameter diagrams reveal only a very limited insight into the dynamics of the system. We therefore use an alternative approach and explore the parameter space by a numerical sampling procedure. Because all parameters in the model have clear interpretation, we can assign a range of realistic values to each of the parameters (see Table 1). We generate an ensemble of parameter sets by randomly assigning each parameter a value drawn from the respective range. The stability of the steady state cor- responding to a sample parameter set is then determined by numerical computation of the eigenvalues of the corresponding Jacobian. Because of the numerical efficiency of eigenvalue computation, ensembles of millions or billions of sample parameter sets can be evaluated in reasonable computational time. Based on such large ensembles, a sound statistical analysis of models containing hundreds or thousands of parameters is feasible. An example of such an analysis in a 50species model was presented in Gross et al. (2009). We refer the reader to this paper for an illustration of the ecological insights that can be gained from correlation analysis in generalized models.

To assess the dependence of the stability of the IGP model on the parameters, we generated $10^{8}$ random parameter sets. Both scale and elasticity parameter values were drawn independently from uniform distributions. Subsequently, each parameter set was assigned a stability value of 1 if it is found to correspond to a stable steady state and 0 if it corresponds to an unstable steady state. The dependence of system stability on individual parameter values was then quantified by computing the correlation coefficient between a given parameter and the stability value over the whole ensemble. Strong positive correlations indicate that large values of the respective parameter promote stability, while strong negative correlations indicate that large values of the parameter reduce stability.

The results of the numerical analysis (Fig. 4) show that the proportional loss of the resource due to intraspecific competition, $\delta$, correlate with stability. This is not surprising because of the known stabilizing effect of super-linear mortality. To a lesser extent high values of $\beta_{x}$ promote stability. This shows that stability is enhanced if the predatory losses of the resource occur
Table 1 Values and ranges of the parameter sampling assumed to compute Fig. 4

The timescales, $\alpha_{x}, \alpha_{y}$ and $\alpha_{z}$, are assumed to scale allometrically. The parameter $r$ describes the timescale separation. The elasticities, $s_{\mathrm{x}}, d_{\mathrm{x}}, m_{\mathrm{y}}, f_{\mathrm{y}}, f_{\mathrm{x}}, k_{\mathrm{t}}$ and $k_{\mathrm{z}}$, are assumed to be one (linear), two (quadratic) or between 0 and 1 (saturation, see text). Only the elasticity $m_{\mathrm{z}}$ is assumed to be slightly super linear. The branching parameters, $\delta, \beta_{x}$ and $\beta_{y}$, are per definition between 0 and 1

\begin{tabular}{lll}
\hline Parameter & Value or range & Meaning \\
\hline$\alpha_{x}$ & 1 & Turnover rate of resource \\
$\alpha_{y}$ & $r$ & Turnover rate of consumer \\
$\alpha_{z}$ & $r^{2}$ & Turnover rate of omnivore \\
$r$ & 0 to 1 & Allometric factor \\
$s_{\mathrm{X}}$ & 1 & Elasticity of resource production \\
$d_{\mathrm{x}}$ & 2 & Elasticity of intraspecific competition in resource \\
$m_{\mathrm{y}}$ & 1 & Elasticity of consumer mortality \\
$m_{\mathrm{z}}$ & 1 & Elasticity of omnivore mortality \\
$f_{\mathrm{y}}$ & 1 & Elasticity of consumption with respect to consumer \\
$f_{\mathrm{X}}$ & 0 to 1 & Elasticity of consumption with respect to resource \\
$k_{\mathrm{t}}$ & 0 to 1 & Elasticity of predation with respect to prey/resource \\
$k_{\mathrm{z}}$ & 1 & Elasticity of predation with respect to omnivore \\
$\delta$ & 0 to 1 & Proportion of losses of the resource due to mortality \\
$\beta_{x}$ & 0 to 1 & Proportion of resource consumption due to consumer \\
$\beta_{y}$ & 0 to 1 & Proportion of losses of consumer due to predation \\
$t_{x}$ & 0 to 1 & Proportion of resource in omnivore diet \\
$t_{y}$ & $1-t_{x}$ & Proportion of consumer in omnivore diet \\
\hline
\end{tabular}




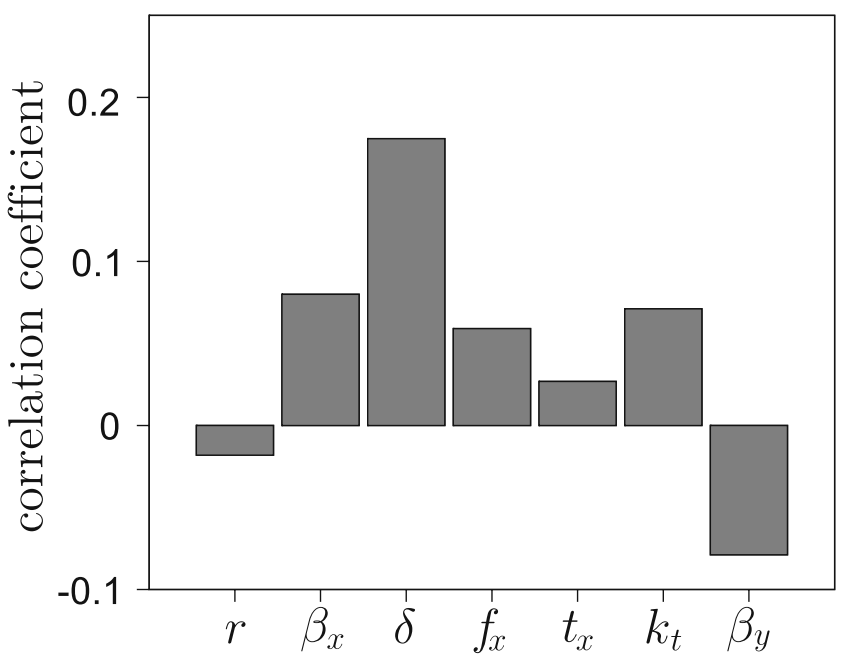

Fig. 4 The dependence of the stability of the generalized IGP model on the parameters: $r, \beta_{\mathrm{x}}, \delta, f_{\mathrm{x}}, t_{\mathrm{x}}, k_{\mathrm{t}}$ and $\beta_{y}$. Dependencies were quantified by calculating the correlation coefficients between a given parameter and system stability. $10^{8}$ parameters sets were assigned randomly from uniform distributions over specified ranges. Error bars are too small to be visible. Strong positive correlations indicate that large values of the specific parameter promote stability, while strong negative correlations indicate that large values of the specific parameter promote instability. The parameters $\delta$, and to a lesser extent $\beta_{x}$, corresponding to high intraspecific competition of $X$ and a strong trophic interaction between $X$ and $Y$, stabilize the generalized IGP model. High values of $\beta_{y}$, strong extrinsic mortality of $Y$, is associated with destabilizing effects. The interpretation of parameters follows the lines explained in Section Density-dependent growth of a single species. Additional information is provided in Table 1

mainly because of predation by the consumer. This is consistent with observation 1 stated in the introduction of this section that stability is contingent on the greater competitive ability of the consumer. Furthermore, Fig. 4 shows a stabilizing effect of $k_{\mathrm{t}}$ and $f_{\mathrm{x}}$, which denote the elasticity of functional responses of the omnivore and the consumer respectively. Already in the previous section we linked such elasticities to enrichment. In enrichment scenarios using common functional forms these parameters tend to decrease leading to instability, which explains observation 2 stated above. We remark, however, that moderate enrichment may have a stabilizing effect if alternative functional forms, such as the one proposed in Gross et al. (2004) are used. The third observation, stated above, is hard to investigate in the generalized model because it refers to abundances in the steady state, which are not directly accessible in the generalized analysis. However, in addition to previous findings the generalized analysis reveals that the fraction of losses of the consumer caused by the omnivore, $\beta_{y}$, is strongly negatively correlated with stability. This indicates that scenarios in which the omnivore benefits from the presence of the consumer are likely to be unstable.

We remark that the precise results of the sampling analysis shown here are not independent of the specific ranges and distributions that are used for generating the ensemble. Although the error bars of the statistical analysis rapidly become very small, minor differences between correlation coefficients should not be over-interpreted. Nevertheless, the stability correlation analysis is a powerful tool that can very quickly convey an impression of the stabilizing and destabilizing factors in large networks. Ideally, this analysis should be followed up by more refined statistical exploration of the ensemble. More detailed insights in the behavior of the system can be gained for instance by plotting histograms of the proportion of stable states that are found if one parameter is set to a specific value, while all others are varied randomly. Such histograms have for instance been used in Steuer et al. (2006), Steuer et al. (2007), Gross et al. (2009) and Zumsande and Gross (2010). Because these more detailed analyses clearly exceed the scope of the present paper, we postpone further analysis of the IGP model to a separate publication.

\section{Conclusions}

In the present paper, we have illustrated the fundamental ideas or procedures of generalized modeling and extended the approach of generalized modeling. Generalized models can reveal conditions for the stability of steady states in large classes of systems, identify the bifurcations in which stability is lost, and provide some insights into the global dynamics of the system. They can be seen as an intermediate approach that has many advantages of conventional equation-based models, while coming close to the efficiency of random matrix models. This efficiency, both in terms of manual labor and CPU time, highlights generalized modeling as a promising approach for detailed analysis of large ecological systems. Although we have restricted the presentation to models with up to three variables, these simple examples already contain all of the complexities that are encountered in larger systems, such as the 50species model studied in Gross et al. (2009).

The presentation of generalized modeling in the present paper differed significantly from previous publications. The differences arise in part from the stronger focus on fundamental concepts and modeling strategies and in part from a newly proposed shortcut that facilitates the formulation of generalized models 
Throughout this paper, we have contrasted several generalized models with conventional counterparts. We emphasize that this was done purely for illustration of the results of generalized modeling. Generalized modeling should by no means be regarded as an alternative modeling approach replacing conventional models. Note that generalized modeling is mainly useful in systems for which little information is available, whereas in well-known systems many more insights may be extractable by conventional models. We point out that the iterative refinement procedure proposed here, allows a researcher to start out with a generalized model and then successively integrate new information as it becomes available until eventually a conventional model is obtained. Generalized modeling is ideally applied if one asks how and under which conditions a given dynamical phenomenon, such as stable coexistence, oscillations or chaos, is possible. Because of its efficiency and generality, generalized modeling can then be used to explore a large range of model structures for evidence pointing to the phenomenon under consideration. Thereby, promising specific model systems can be identified which can subsequently be analyzed in more detail by conventional model analysis.

Open Access This article is distributed under the terms of the Creative Commons Attribution Noncommercial License which permits any noncommercial use, distribution, and reproduction in any medium, provided the original author(s) and source are credited.

\section{Appendix 1}

The formulation of elasticity parameters is contingent on the relationship

$$
\left.\frac{\partial F}{\partial X}\right|_{*}=\left.\frac{F^{*}}{X^{*}} \frac{\partial \log F}{\partial \log X}\right|_{*}
$$

where $F$ represents some function of $X$. For proving this relationship, we consider the right-hand side and multiply it by $1=\partial F / \partial F$

$$
\left.\frac{F^{*}}{X^{*}} \frac{\partial \log F}{\partial F} \frac{\partial F}{\partial \log X}\right|_{*}
$$

where $(\partial \log F / \partial F)_{*}$ simplifies to $1 / F^{*}$. This results in

$$
\left.\frac{\partial F}{\partial X}\right|_{*}=\left.\frac{1}{X^{*}} \frac{\partial F}{\partial \log X}\right|_{*} .
$$

In the previous steps, we replaced $\log F$ in the numerator of the derivative. To replace $\log X$ in the numerator we proceed analogously

$$
\left.\frac{\partial F}{\partial X}\right|_{*}=\left.\frac{1}{X^{*}} \frac{\partial X}{\partial \log X} \frac{\partial F}{\partial X}\right|_{*}
$$

We now consider the second factor of the right-hand side. To evaluate the partial derivative we define $X=$ $\mathrm{e}^{u}$ and write

$$
\left.\frac{\partial X}{\partial \log X}\right|_{*}=\left.\frac{\partial \mathrm{e}^{u}}{\partial \log \mathrm{e}^{u}}\right|_{*}=\left.\frac{\partial \mathrm{e}^{u}}{\partial u}\right|_{*}=\left.\mathrm{e}^{u}\right|_{*}=X^{*}
$$

substituting back into Eq. 47 we obtain

$$
\left.\frac{\partial F}{\partial X^{*}}\right|_{*}=\left.\frac{1}{X^{*}} X^{*} \frac{\partial F}{\partial X}\right|_{*}=\left.\frac{\partial F}{\partial X}\right|_{*} .
$$

which proves Eq. 44.

\section{Appendix 2}

Starting from

$G(T(X, Y), Z)=\frac{\epsilon_{g} T_{X} X}{T(X, Y)} K(T(X, Y), Z)$

the elasticity with respect to $X$ can be subsequently be formulated as

$$
\begin{aligned}
g_{\mathrm{x}}= & \frac{\partial \log G}{\partial \log X} \\
= & \frac{X^{*}}{G^{*}} \frac{\partial}{\partial X} \frac{\epsilon_{g} T_{x} X}{T(X, Y)} K(T(X, Y), Z) \\
= & \frac{\epsilon_{g} X^{*} T^{*}}{\epsilon_{g} T_{x} X^{*} K^{*}}\left(\frac{T_{x} K^{*}}{T^{*}}+\frac{T_{x} X^{*}}{T^{*}} \frac{\partial K}{\partial X}\right. \\
& \left.\quad+T_{x} X^{*} K^{*} \frac{\partial}{\partial X} \frac{1}{T(X, Y)}\right) \\
= & \frac{T^{*}}{T_{x} K^{*}}\left(\frac{T_{x} K^{*}}{T^{*}}+\frac{T_{x} K^{*}}{T^{*}} \frac{X^{*}}{K^{*}}\right. \\
& \left.\quad \times \frac{\partial K}{\partial X}+\frac{T_{x} K^{*}}{T^{*}} T^{*} X^{*}\left(-\frac{1}{\left(T^{*}\right)^{2}} \frac{\partial T}{\partial X}\right)\right) \\
= & 1+\frac{T^{*}}{K^{*}} \frac{\partial K}{\partial T} \frac{X^{*}}{T^{*}} \frac{\partial T}{\partial X}-\frac{X^{*}}{T^{*}} \frac{\partial T}{\partial X} \\
= & 1+k_{\mathrm{t}} t_{\mathrm{x}}-t_{\mathrm{x}} \\
= & k_{\mathrm{t}} t_{\mathrm{x}}+t_{\mathrm{y}} .
\end{aligned}
$$

\section{References}

Bascompte J, Melián C, Sala E (2005) Interaction strength combinations and the overfishing of a marine food web. Proc Natl Acad Sci U S A 102:5443-5447

Baurmann M, Gross T, Feudel U (2007) Instabilities in spatially extended predator-prey systems: spatio-temporal patterns in the neighborhood of turing-hopf bifurcations. J Theor Biol 245:220-229

Berryman AA (1981) Population systems: a general introduction. Population systems: a general introduction. Plenum

DeAngelis DL, Goldstein RA, O’Neill RV (1975) A model for tropic interaction. Ecology 56:881-892 
Diehl S, Feissel M (2001) Intraguild prey suffer from enrichment of their resources: a microcosm experiment with ciliates. Ecology 82(11):2977-2983

Fell DA, Sauro HM (1985) Metabolic control and its analysisadditional relationships between elasticities and control coefficients. Eur J Biochem 148(3):555-561

Fryxell JM, Mosser A, Sinclair ARE, Packer C (2007) Group formation stabilizes predator-prey dynamics. Nature 449(7165):1041-1043

Gardner MR, Ashby WR (1970) Connectance of large dynamic (cybernetic) systems-critical values for stability. Nature 228(5273):784

Getz W (1984) Population dynamics: a per capita resource approach. J Theor Biol 108:623-643

Gross T, Ebenhöh W, Feudel U (2004) Enrichment and foodchain stability: the impact of different forms of predatorprey interaction. J Theor Biol 227:349-358

Gross T, Feudel U (2004) Analytical search for bifurcation surfaces in parameter space. Physica D 195(3-4):292-302

Gross T, Ebenhoh W, Feudel U (2005) Long food chains are in general chaotic. Oikos 109:1-10

Gross T, Feudel U (2006) Generalized models as a universal approach to the analysis of nonlinear dynamical systems. Phys Rev E 73:016205

Gross T, Feudel U (2009) Local dynamical equivalence of certain food webs. Ocean Dyn 59:417-427

Gross T, Feudel U (2009) The invisible niche: weakly densitydependent mortality and the coexistence of species $\mathrm{J}$ Theor Biol 258:148-155

Gross T, Rudolf L, Levin SA, Dieckmann U (2009) Generalized models reveal stabilizing factors in food webs. Science 325(5941):747-750

Guckenheimer J, Holmes P (2002) Nonlinear oscillations, dynamical systems, and bifurcations of vector fields. Springer, Berlin

Holling CS (1959) Some characteristics of simple types of predation and parasitism. Can Entomol 91:385-389

Holt RD, Polis GA (1997) A theoretical framework for intraguild predation. Am Nat 149:745-764

Jamshidi N, Palsson BO (2008) Formulating genome-scale kinetic models in the post-genome era. Molecular Systems Biology 4:171

Kuijper L, Kooi B, Zonneveld C, Kooijman S (2003) Omnivory and food web dynamics. Ecol Model 163(1-2):19-32

Kuznetsov YA (2004) Elements of applied bifurcation theory. Springer, Berlin

Levin SA (1977) A more functional response to predator-prey stability. Am Nat 111(978):381-383

May RM (1972) Will a large complex system be stable. Nature 238(5364):413-414

McCann K, Hastings A (1997) Re-evaluating the omnivorystability relationship in food webs. Proc R Soc B Biol Sci 264(1385):1249

Murdoch W, Oaten A (1975) Predation and population stability. In: Cragg JB, Macfadyen A (eds) Advances in ecological research, vol 9. Academic, New York. pp 1-131

Murdoch WW (1977) Stabilizing effects of spatial heterogeneity in predator-prey systems. Theor Popul Biol 11(2):252-273
Namba T, Tanabe K, Maeda N (2008) Omnivory and stability of food webs. Ecological Complexity 5(2):73-85

Pimm S, Lawton J (1978) On feeding on more than one trophic level. Nature 275:542-544

Polis G (1991) Complex trophic interactions in deserts-an empirical critique of food-web theory. Am Nat 138(1):123-155

Reznik E, Segrè D (2010) On the stability of metabolic cycles. J Theor Biol 266(4):536-549

Rodriguez A, Infante D (2009) Network models in the study of metabolism. Electron J Biotechnol 12(4):1-19

Rosenzweig ML (1971) Paradox of enrichment: destabilization of exploitation ecosystems in ecological time. Science 171:385387

Rosenzweig ML, MacArthur RH (1963) Graphical representation and stability conditions of predator-prey interactions. Am Nat 97:209-223

Schallau K, Junker BH (2010) Simulating plant metabolic pathways with enzyme-kinetic models. Plant Physiol 152(4): 1763-1771

Steuer R (2007) Computational approaches to the topology, stability and dynamics of metabolic networks. Phytochemistry 68(16-18):2139-2151

Steuer R, Gross T, Selbig J, Blasius B (2006) Structural kinetic modeling of metabolic networks. Proc Natl Acad Sci 103:11,868-11,873

Steuer R, Junker BH (2009) Computational models of metabolism: stability and regulation in metabolic networks. Adv Chem Phys 142:105-251

Steuer R, Nesi AN, Fernie AR, Gross T, Blasius B, Selbig J (2007) From structure to dynamics of metabolic pathways: application to the plant mitochondrial tca cycle. Bioinformatics 23:1378-1385

Stiefs D, Gross T, Steuer R, Feudel U (2008) Computation and visualization of bifurcation surfaces. Int $\mathrm{J}$ Bifurc Chaos 18:2191-2206

Stiefs D, van Voorn GAK, Kooi BW, Gross T, Feudel U (2010) Food quality in produce-grazer models- a generalized analysis. Am Nat 176:367-380

Stouffer DB, Bascompte J (2010) Understanding food-web persistence from local to global scales. Ecol Lett 13(2):154 161

Sweetlove LJ, Fell D, Fernie AR (2008) Getting to grips with the plant metabolic network. Biochem J 409(Part 1):27-41

Tanabe K, Namba T (2008) Omnivory creates chaos in simple food web models. Ecology 86:3411-3414

Verdy A, Amarasekare P (2010) Alternative stable states in communities with intraguild predation. J Theor Biol 262(1):116128

van Voorn GAK, Hemerik L, Boer MP, Kooi BW (2007) Heteroclinic orbits indicate overexploitation in predator-prey systems with a strong allee effect. Math Biosci 2009:451469

Wollkind D, Hastings A, Logan J (1982) Age structure in predator-prey systems. II. functional response and stability and the paradox of enrichment. Theor Popul Biol 21(1):5768

Zumsande M, Gross T (2010) Bifurcations and chaos in the MAPK signaling cascade. J Theor Biol 265(3):481-491 